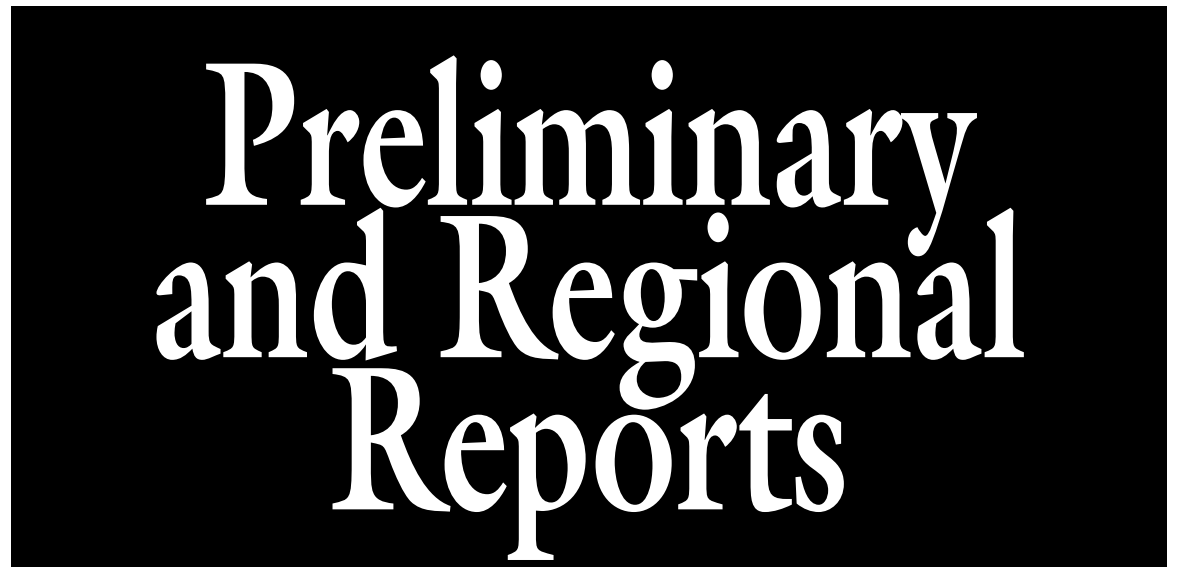

\section{Effects of Nitrogen Fertilization Rate on Aesthetic Quality of Landscape-grown Annuals and Perennials}

\author{
Kimberly A. Moore ${ }^{1,7,11}$, Amy L. Shober ${ }^{2,8}$, Gitta S. Hasing ${ }^{3,9}$, \\ Christine L. Wiese ${ }^{4,9}$, Geoffrey C. Denny ${ }^{5,10}$, and Gary W. Knox ${ }^{6,7}$
}

ADDITIONAL INDEX WORDs. best management practices, landscape maintenance, ornamental plants

SUMMARY. Recent research suggested that the nitrogen $(\mathrm{N})$ fertilizer rates needed to maintain high-quality landscape plants was lower than rates needed to grow the largest size plants. Our objective was to evaluate the effect of $\mathbf{N}$ fertilizer rate on the aesthetic quality of various landscape-grown annual and perennials species. Nineteen cool-season annuals, 20 warm-season annuals, and 4 perennials were planted into raised beds containing subsoil fill material in a completely randomized design in west-central Florida (U.S. Department of Agriculture hardiness zone 9b). Plants were fertilized every 12 weeks with polymer coated, slow-release $\mathrm{N}(42 \mathrm{~N}-0 \mathrm{P}-0 \mathrm{~K})$ fertilizer at annual $\mathrm{N}$ rate of 3,5 , or $7 \mathrm{lb} / 1000 \mathrm{ft}^{2}$ (annuals) or 1,3 , or $5 \mathrm{lb} / 1000 \mathrm{ft}^{2}$ (perennials). Plants were rated for aesthetic quality every 6 weeks for a period of 18 weeks (annuals) or 54 weeks (perennials). For most species, quality ratings of plants fertilized with $3 \mathrm{lb} / 1000 \mathrm{ft}^{2}$ of $\mathrm{N}$ per year (annuals) or $1 \mathrm{lb} / 1000 \mathrm{ft}^{2}$ of $\mathrm{N}$ per year (perennials) were not significantly lower than plants receiving higher rates of $\mathrm{N}$ annually. Previously reported $\mathrm{N}$ fertilizer recommendations for central Florida of 2 to $4 \mathrm{lb} / 1000 \mathrm{ft}^{2}$ per year should be adequate for maintaining acceptable quality landscape-grown annual and herbaceous perennial plant species.

$\mathrm{B}$ etween $40 \%$ and $60 \%$ of nitrogen that is applied to plants in fertilizers is actually taken up by plant roots; the remainder stays in the soil or moves offsite via leaching, runoff, or volatilization (Matson et al., 1997). Therefore, efficient application of fertilizers is necessary to prevent losses of $\mathrm{N}$ to the environment (Latimer et al., 1996). Careful management of fertilizer application rate, timing, and method of application is required to ensure that fertilizer application does not exceed the specific $\mathrm{N}$ requirements of growing plants (Line et al., 2002). Both homeowners and landscape professionals can contribute to the improvement of water quality by fertilizing landscapes at the minimum required levels for healthy, attractive plants. However, few studies evaluated how much fertilizer is needed to maintain aesthetically pleasing plants in the landscape (Shurberg et al., 2012a, b).

The University of Florida-Institute of Food and Agricultural Sciences (UFIFAS) Florida Friendly Landscaping ${ }^{\mathrm{TM}}$ Program (FFL) provides general fertilizer recommendations for ornamental landscape plants ranging from 0 to $6 \mathrm{lb} /$ $1000 \mathrm{ft}^{2}$ of N (FFL, 2009). Shurberg et al. $(2012 \mathrm{a}, \mathrm{b})$ evaluated the growth and quality of a limited number of annual and perennial species in central Florida with $\mathrm{N}$ applications of $0-12$ $\mathrm{lb} / 1000 \mathrm{ft}^{2}$. They suggested that attractive annual and perennial ornamental plant species could be maintained in the Florida landscape with relatively low $\mathrm{N}$ inputs (2 to $4 \mathrm{lb} /$ $1000 \mathrm{ft}^{2}$ for annuals and 4 to $6 \mathrm{lb} /$ $1000 \mathrm{ft}^{2}$ for perennials), provided the goal was to produce high-quality plants rather than plants of maximum size. However, the research was limited to the evaluation of the plant quality response to $\mathrm{N}$ fertilizer rate for only a few species of annual and perennial landscape plants. Screening of additional plants is necessary to determine if $\mathrm{N}$ fertilizer rates recommended by Shurberg et al. $(2012 \mathrm{a}, \mathrm{b})$ can be applied broadly to other landscapegrown annual and perennial ornamental species in Florida. The objective of this study was to evaluate the quality response of a broad spectrum of landscape annuals and perennials to $\mathrm{N}$ fertilizer applied at three rates to confirm that highquality plants can be produced at $\mathrm{N}$ rates recommended by Shurberg et al. $(2012 a, b)$.

\section{Materials and methods}

Plant material. A total of 35 annual (cool- and warm-season) annuals species were selected for validation of $\mathrm{N}$ recommendations over two growing seasons (Table 1 ). In addition, 'Montego Yellow' or 'Montego Purple' snapdragon (Antirrbimum majus) 
and 'Profusion Cherry' zinnia (Zinnia elegans $\times$ angustifolia), which are cooland warm-season annual species evaluated previously by Shurberg et al. (2012b), were planted as reference species in both years to ensure a consistent quality response to the $\mathrm{N}$ fertilizer rates across studies. Annual plant species were received as plugs from Knox Nursery (Winter Garden, FL), as seed from Park Seed (Greenwood, SC) or Harris Seed (Rochester, NY), or in 4inch pots from American Farms (Naples, FL). Cool- and warm-season plugs and seeds were grown in 4-inch azalea pots (Reb Plastic Inc., Orlando, FL) for $\approx 6$ (plugs) or 8 weeks (seed) before transplanting into raised beds, to ensure that all plants were of comparable size at transplanting. To maximize consistency in $\mathrm{N}$ contributions during rearing, annual species were fertilized with a liquid $21 \mathrm{~N}-3.15 \mathrm{P}-$ $5.8 \mathrm{~K}$ acid special fertilizer (Peter's Professional ${ }^{\circledR}$; Everris NA, Dublin, $\mathrm{OH}$ ) at an $\mathrm{N}$ rate of $100 \mathrm{ppm}$ twice per week for 1 week, then at an $\mathrm{N}$ rate of $200 \mathrm{ppm}$ twice weekly until transplanting.

Three herbaceous perennials [blanket flower (Gaillardia pulchella), goldenrod (Solidago chapmanii), and mondo grass (Ophiopogon japonicas)] were also selected for validation of $\mathrm{N}$ rate recommendations. 'Mystic Spires' salvia (Salvia

\footnotetext{
The work was supported by the Florida Agricultural Experiment Station, the Southwest Florida Water Management District, and the Florida Nursery Growers and Landscape Association.

${ }^{1}$ Fort Lauderdale Research and Education Center, Environmental Horticulture Department, Institute of Food and Agriculture Science, University of Florida, 3205 College Avenue, Davie, FL 33314

${ }^{2}$ Department of Plant and Soil Sciences, University of Delaware, 531 South College Avenue, Newark, DE 19716

${ }^{3}$ Gulf Coast Research and Education Center, Environmental Horticulture Department, Institute of Food and Agriculture Science, University of Florida, 14625 CR 672, Wimauma, FL 33598

${ }^{4}$ Environmental Horticulture Department, Institute of Food and Agriculture Science, University of Florida, 106 Building 68, Gainesville, FL 32611

${ }^{5}$ Mississippi State University, 246 Dorman Hall, Mississippi State, MS 39762

${ }^{6}$ North Florida Research and Education Center, Environmental Horticulture Department, Institute of Food and Agriculture Science, University of Florida, 155 Research Road, Quincy, FL 32351

${ }^{7}$ Professor

${ }^{8}$ Associate Professor

${ }^{9}$ Biologist

${ }^{10}$ Assistant Extension Professor

${ }^{11}$ Corresponding author. E-mail: klock@ufl.edu.
}

longispicata $\times$ farinacea) was evaluated previously by Shurberg et al. (2012a) and was planted as a reference species to ensure a consistent quality response to the $\mathrm{N}$ fertilizer rates across studies. All perennial species were received in 1-gallon containers; blanket flower and goldenrod were received from Sweetbay Nursery (Parrish, FL); mondo grass and salvia were received from Landscape Flower Growers, Inc. (Riverview, FL).

EXPERIMENTAL DESIGN. Raised beds $(10 \times 50 \times 0.5 \mathrm{ft})$ containing subsoil fill material (St. John's fine sand, Sandy, siliceous, hyperthermic Typic Alaquods) [U.S. Department of Agriculture (USDA), 2004] were constructed at the UF-IFAS Gulf Coast Research and Education Center in Wimauma, FL (USDA hardiness zone $9 b$ ). An initial sample of soil fill was analyzed for $\mathrm{pH}$ and soil nutrient content before transplanting as reported previously in our studies comparing annual and perennial growth to $\mathrm{N}$ fertilization under similar growing conditions in central Florida (Shurberg et al., 2012a, b). Sixteen annual plants of each species were planted in a square configuration (four plant species $\times$ four replicate plants spaced $1 \mathrm{ft}$ apart) in a total of nine raised beds (plots). Coolseason annuals species were planted into raised beds on 29 Oct. 2010 (year 1) and 6 Dec. 2011 (year 2). Warm-season annual species were planted into raised beds on 25 May 2011 (year 1) and 3 May 2012 (year 2 ). Fifteen herbaceous perennial plants of each species were planted in a rectangle configuration (five plant species $\times$ three plant replicates spaced $1 \mathrm{ft}$ apart) in the remaining area within the same nine plots on 4 Mar. 2011.

Irrigation was applied through nine drip lines (Jain Irrigation, Winter Haven, FL) installed on $\mathrm{l}-\mathrm{ft}$ spacing with 8 -inch spacing between emitters and a flow rate of $0.65 \mathrm{gal} / 100 \mathrm{ft}^{2}$ per minute. Plants were irrigated for $25 \mathrm{~min}$, three times per week starting at $0830 \mathrm{HR}$. Cumulative rainfall was 82.6 inches from May 2010 to Apr. 2012 with average temperatures ranging from 51 to $81^{\circ} \mathrm{F}$, maximum temperatures of $96^{\circ} \mathrm{F}$, and minimum temperatures of $22{ }^{\circ} \mathrm{F}$ (University of Florida, 2014). Weeds were manually removed or treated with glyphosate (Round-Up ${ }^{\circledR} ;$ Monsanto, Creve Coeur, MO) as needed. No mulch was added to beds to minimize outside $\mathrm{N}$ contributions. All plant species were fertilized at three different $\mathrm{N}$ rates, not to exceed current recommendations for landscape fertilization (Florida Department of Environmental Protection, 2002). Plants were fertilized every 12 weeks (based on the release rate of the fertilizer published by the manufacturer) with a $42 \mathrm{~N}-0 \mathrm{P}-$ 0K polymer-coated, controlled-release N source (Polyon; Harrell's, Lakeland, $\mathrm{FL}$ ) at the following annual $\mathrm{N}$ rates: 3 , 5 , or $7 \mathrm{lb} / 1000 \mathrm{ft}^{2}$ (annuals) and 1,3 , or $5 \mathrm{lb} / 1000 \mathrm{ft}^{2}$ (perennials).

Plant aesthetic Quality RATINGs. Aesthetic quality ratings $(0-5)$, which considered canopy density, canopy dieback, flowers, and general form, were assigned every 6 weeks to the middle plants (four annuals and three perennials) from each planting to avoid potential edge effects. Quality ratings were applied on the following scale: $0=$ dead, $1=$ poor (low canopy density, few to no flowers, and/or substantial chlorosis), 2 = below average (significant dieback or chlorosis, lower density, and flowering), 3 = average or acceptable (moderate dieback, limited chlorosis, adequate form and flowering), $4=$ above average (minimal dieback, healthy plant, above average density and form), and $5=$ outstanding (dense leaf canopy, high-quality flowers, no nutrient deficiencies or dieback) (Shober et al., 2009). Annual species were evaluated for 18 weeks and perennial species were evaluated for 54 weeks.

Statistical analysis. The experiments were designed as a completely randomized design, with $\mathrm{N}$ rates applied randomly over nine raised bed plots containing either four (annuals) or three (perennials) plants of each species per bed. Each plant type (cool-season annuals, warm-season annuals, herbaceous perennials) and species was evaluated separately. Plant aesthetic quality data for all plant species was analyzed using PROC GLIMMIX (SAS version 9.2; SAS Institute, Cary, NC) by species and weeks after planting (WAP), with $\mathrm{N}$ rate as a fixed effect. Plant cluster $\times$ treatment was included as a random effect using a normal distribution and an identity link function. No planting 
Table 1. Common name, scientific name, cultivar, and source of cool and warm-season annual plant materials used to evaluate aesthetic quality response to nitrogen $(\mathrm{N})$ fertilizer rate for landscape-grown plants in west-central Florida (U.S. Department of Agriculture hardiness zone 9b).

\begin{tabular}{|c|c|c|c|c|}
\hline Plant type and planting date & Common name & Scientific name & Cultivar & Source $^{\mathrm{z}}$ \\
\hline \multicolumn{5}{|l|}{ Cool-season annuals } \\
\hline \multicolumn{5}{|l|}{29 Oct. 2010} \\
\hline & Alyssum & Lobularia maritima & $\mathrm{N} / \mathrm{A}^{\mathrm{y}}$ & Knox nursery \\
\hline & Dahlberg daisy & Thymophylla tenuiloba & $\mathrm{N} / \mathrm{A}$ & Knox nursery \\
\hline & Geranium & Pelargonium $\times$ hortorum & Survivor Hot Pink & American Nursery \\
\hline & Gomphrena & Gomphrena globosa & $\mathrm{N} / \mathrm{A}$ & Knox nursery \\
\hline & Impatiens & Impatiens walleriana & $\begin{array}{l}\text { Super Elfin Bright } \\
\text { Orange }\end{array}$ & American nursery \\
\hline & Linaria & Linaria sp. & Enchantment & Knox nursery \\
\hline & Petunia & Petunia $\times$ bybrida & Plush Salmon & Knox nursery \\
\hline & Phlox & Phlox drummondii & 21st Century Scarlet & Knox nursery \\
\hline & Wax begonia & $\begin{array}{l}\text { Begonia } \times \text { semperflorens- } \\
\text { cultorum }\end{array}$ & Bada Bing White & American nursery \\
\hline \multicolumn{5}{|l|}{6 Dec. 2011} \\
\hline & Flowering cabbage & Brassica oleracea & Pigeon Purple & Harris seed \\
\hline & Foxglove & Digitalis purpurea & Camelot Lavender & Park seed \\
\hline & Gypsophila & Gypsophila muralis & Gypsy Deep Rose & Harris seed \\
\hline & Ornamental kale & Brassica oleracea & Kamome White & American farms \\
\hline & Nasturtium & Tropaeolum nanum & Princess of India & Harris seed \\
\hline & Shasta daisy & Leucanthemum $\times$ superbum & White Breeze & Park seed \\
\hline & Statice & Limonium sinuatum & Fortress Dark Blue & Harris seed \\
\hline & Viola & Viola cornuta & Penny Blue & Knox nursery \\
\hline \multicolumn{5}{|l|}{ Reference species ${ }^{\mathrm{x}}$} \\
\hline & Snapdragon & Antirrbinum majus & $\begin{array}{l}\text { Montego Yellow; } \\
\text { Montego Purple }\end{array}$ & Knox nursery \\
\hline \multicolumn{5}{|l|}{ Warm-season annuals } \\
\hline & Ageratum & Ageratum houstonianum & $\begin{array}{l}\text { Blue Puffs Improved } \\
\text { (Blue Danube) }\end{array}$ & Knox nursery \\
\hline & Cosmos & Cosmos sulphureus & $\begin{array}{l}\text { Cosmic Mix; Cosmic } \\
\text { Orange }\end{array}$ & Knox nursery \\
\hline & Dusty miller & Senecio cineraria & Silverdust & Knox nursery \\
\hline & Lobelia & Lobelia erinus & Riverine Sky Blue & Knox nursery \\
\hline & Marigold & Tagetes patula & Bonanza Orange & Knox nursery \\
\hline & Moss rose & Portulaca grandiflora & Sundial Yellow & Knox nursery \\
\hline & Nicotiana & Nicotiana alata & Saratoga Red & Knox nursery \\
\hline & Salvia & Salvia splendens & Vista Red & Knox nursery \\
\hline & Torenia & Torenia fournieri & Crown Plum & Knox nursery \\
\hline \multicolumn{5}{|l|}{3 May 2012} \\
\hline & African daisy & Osteospermum asti & Lavender Shades $^{\mathrm{TM}}$ & Park seed \\
\hline & Angelonia & Angelonia angustifolia serena & Lavender Pink $k^{\top M}$ & Park seed \\
\hline & Balloon flower & Platycodon grandiflorus & Komachi & Park seed \\
\hline & Celosia & Celosia spicata & Spiky Purple & Harris seed \\
\hline & Gazania & Gazania splendens & Kiss Orange Flame & Harris seed \\
\hline & Lavender & Lavandula angustifolia & Lavender Lady & Park seed \\
\hline & Nierembergia & Nierembergia hippomanica & White Robe & Harris seed \\
\hline & Ornamental basil & Ocimum basilicum & Cardinal Organic & Harris seed \\
\hline & Ornamental pepper & Capsicum annuиm & Medusa & Harris seed \\
\hline Kererence species & Zinnia & Zinnia elegans $\times$ angustifolia & Profusion cherry & Knox nursery \\
\hline
\end{tabular}

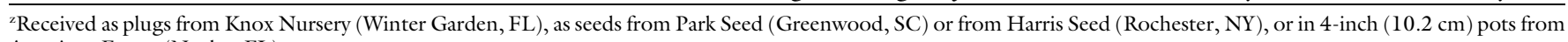
American Farms (Naples, FL).

${ }^{\mathrm{N}} \mathrm{/A}$ indicates no plant cultivar.

${ }^{x}$ Reference species indicates species that were also evaluated by Shurberg et al. (2012a, b).

year $\times \mathrm{N}$ rate interaction was noted for annuals reference-species quality; therefore quality data from both years were combined for analysis.
Pairwise comparisons for aesthetic quality were conducted with the Tukey's honestly significant difference test at a significance level of $\alpha=0.05$. Data were checked for normality by examining histogram and normality plots of the conditional residuals. 


\section{Results and discussion}

COOL-ANDWARM-SEASONANNUALS. Previous research at the central Florida experiment location evaluated the growth responses of cool and warm-season annuals to $\mathrm{N}$ fertilizer rate (Shurberg et al., 2012b). However, the goal of this research was to validate the suggested $\mathrm{N}$ fertilization rates to maintain acceptable quality cool and warm-season annuals. Aesthetic quality snapdragon, foxglove (Digitalis purpurea), and kale (Brassica oleracea) were affected by fertilizer rate (Table 2). Higher quality ratings were assigned to snapdragon receiving $7 \mathrm{lb} / 1000 \mathrm{ft}^{2}$ per year $\mathrm{N}$ compared with plants receiving 3 or $5 \mathrm{lb} / 1000 \mathrm{ft}^{2} \mathrm{~N}$ per year at 6 WAP and $3 \mathrm{lb} / 1000 \mathrm{ft}^{2} \mathrm{~N}$ per year 12 WAP. However, snapdragon consistently received quality ratings of 3 or greater (all weeks in both years) regardless of $\mathrm{N}$ fertilizer rate (Table 2 ). At $6 \mathrm{WAP}$, foxglove had higher quality when fertilized with $\mathrm{N}$ at the $7 \mathrm{lb} /$ $1000 \mathrm{ft}^{2}$ per year rate compared with plants that received $\mathrm{N}$ at an annual rate of $3 \mathrm{lb} / 1000 \mathrm{ft}^{2}$. However, no further fertilizer rate effects were noted for foxglove quality; all fertilizer rates produced foxglove plants with a quality rating of 3 or greater (Table 2). Aesthetic quality of kale was greater when plants were fertilized with $\mathrm{N}$ at an annual rate of 5 and $7 \mathrm{lb} / 1000 \mathrm{ft}^{2}$ at $12 \mathrm{WAP}$ and $7 \mathrm{lb} /$ $1000 \mathrm{ft}^{2}$ at 18 WAP than when plants received only $3 \mathrm{lb} / 1000 \mathrm{ft}^{2}$ per year $\mathrm{N}$ (Table 2). There were no effects of $\mathrm{N}$ rate on aesthetic quality for any other cool-season annual species. Aesthetic quality ratings for cool-season annual receiving $3 \mathrm{lb} / 1000 \mathrm{ft}^{2} \mathrm{~N}$ per year ranged from 0.0 to 4.8 (Table 2 ). The quality of several cool-season annual species at 18 WAP [e.g., gypsophila (Gypsophila muralis), impatiens (Impatiens wallerana), ornamental kale, linaria (Linaria sp.), nasturtium (Tropaedum nanum), and statice (Limonium sinuatum)] was below average, regardless of $\mathrm{N}$ fertilizer rates; all gomphrena (Gomphrena globosa) were dead by 12 WAP (Table 2 ). We suspect that environmental conditions, cultivar selection, or plant age may have impacted growth. Nitrogen fertilizer rate did not appear to be the major contributing factor to the low quality or death of these cool-season annual species.
Table 2. Plant quality response of selected landscape-grown cool season annual species to nitrogen $(\mathrm{N})$ rate in 2 years in west-central Florida (U.S. Department of Agriculture hardiness zone 9b).

\begin{tabular}{|c|c|c|c|}
\hline \multirow{2}{*}{$\begin{array}{l}\text { Species and fertilizer } \mathrm{N} \text { rate } \\
\left(\mathrm{lb} / 1000 \mathrm{ft}^{2}\right)^{\mathrm{z}}\end{array}$} & \multicolumn{3}{|c|}{ Plant aesthetic quality $(0-5 \text { scale })^{\mathrm{y}}$} \\
\hline & $6 \mathrm{WAP}^{\mathrm{x}}$ & $12 \mathrm{WAP}$ & $18 \mathrm{WAP}$ \\
\hline \multicolumn{4}{|l|}{ Alyssum } \\
\hline 3 & $2.9 \mathrm{a}^{\mathrm{w}}$ & $3.6 \mathrm{a}$ & $3.2 \mathrm{a}$ \\
\hline 5 & $3.6 \mathrm{a}$ & $3.8 \mathrm{a}$ & $3.6 \mathrm{a}$ \\
\hline 7 & $3.6 \mathrm{a}$ & $3.8 \mathrm{a}$ & $3.3 \mathrm{a}$ \\
\hline \multicolumn{4}{|l|}{ 'Bada Bing White' begonia } \\
\hline 3 & $一^{\mathrm{v}}$ & $2.9 \mathrm{a}$ & $3.4 \mathrm{a}$ \\
\hline 5 & - & $3.5 \mathrm{a}$ & $3.8 \mathrm{a}$ \\
\hline 7 & - & $3.4 \mathrm{a}$ & $4.3 \mathrm{a}$ \\
\hline \multicolumn{4}{|l|}{ Dahlberg daisy } \\
\hline 3 & $3.0 \mathrm{a}$ & $2.6 \mathrm{a}$ & $3.4 \mathrm{a}$ \\
\hline 5 & $3.3 \mathrm{a}$ & $2.9 \mathrm{a}$ & $3.9 \mathrm{a}$ \\
\hline 7 & $3.6 \mathrm{a}$ & $3.4 \mathrm{a}$ & $3.7 \mathrm{a}$ \\
\hline \multicolumn{4}{|l|}{ 'Camelot Lavender' foxglove } \\
\hline 3 & $3.7 \mathrm{~b}$ & $3.5 \mathrm{a}$ & $3.3 \mathrm{a}$ \\
\hline 5 & $3.9 \mathrm{ab}$ & $3.8 \mathrm{a}$ & $3.8 \mathrm{a}$ \\
\hline 7 & $4.0 \mathrm{a}$ & $4.1 \mathrm{a}$ & $4.2 \mathrm{a}$ \\
\hline \multicolumn{4}{|c|}{ 'Pigeon Purple' flowering cabbage } \\
\hline 3 & $4.6 \mathrm{a}$ & $3.8 \mathrm{a}$ & $3.2 \mathrm{a}$ \\
\hline 5 & $4.3 \mathrm{a}$ & $4.0 \mathrm{a}$ & $3.1 \mathrm{a}$ \\
\hline 7 & $4.8 \mathrm{a}$ & $4.3 \mathrm{a}$ & $3.3 \mathrm{a}$ \\
\hline \multicolumn{4}{|l|}{ 'Survivor Hot Pink' geranium } \\
\hline 3 & $1.9 \mathrm{a}$ & $3.1 \mathrm{a}$ & $2.8 \mathrm{a}$ \\
\hline 5 & $1.9 \mathrm{a}$ & $3.3 \mathrm{a}$ & $3.2 \mathrm{a}$ \\
\hline 7 & $2.3 \mathrm{a}$ & $3.7 \mathrm{a}$ & $3.5 \mathrm{a}$ \\
\hline \multicolumn{4}{|l|}{ Gomphrena } \\
\hline 3 & $3.8 \mathrm{a}$ & $0.0 \mathrm{a}$ & $0.0 \mathrm{a}$ \\
\hline 5 & $3.9 \mathrm{a}$ & $0.0 \mathrm{a}$ & $0.0 \mathrm{a}$ \\
\hline 7 & $3.9 \mathrm{a}$ & $0.0 \mathrm{a}$ & $0.0 \mathrm{a}$ \\
\hline \multicolumn{4}{|l|}{ 'Gypsy Deep Rose' gypsophila } \\
\hline 3 & $4.8 \mathrm{a}$ & $4.1 \mathrm{a}$ & $2.6 \mathrm{a}$ \\
\hline 5 & $4.9 \mathrm{a}$ & $4.5 \mathrm{a}$ & $2.2 \mathrm{a}$ \\
\hline 7 & $4.8 \mathrm{a}$ & $4.3 \mathrm{a}$ & $2.2 \mathrm{a}$ \\
\hline \multicolumn{4}{|c|}{ 'Super Elfin Bright Orange' impatiens } \\
\hline 3 & - & $2.5 \mathrm{a}$ & $2.5 \mathrm{a}$ \\
\hline 5 & - & $3.2 \mathrm{a}$ & $2.5 \mathrm{a}$ \\
\hline 7 & - & $2.2 \mathrm{a}$ & $2.1 \mathrm{a}$ \\
\hline \multicolumn{4}{|l|}{ 'Enchantment' linaria } \\
\hline 3 & $3.5 \mathrm{a}$ & $3.7 \mathrm{a}$ & $2.6 \mathrm{a}$ \\
\hline 5 & $3.8 \mathrm{a}$ & $3.8 \mathrm{a}$ & $2.8 \mathrm{a}$ \\
\hline 7 & $4.0 \mathrm{a}$ & $3.9 \mathrm{a}$ & $2.6 \mathrm{a}$ \\
\hline \multicolumn{4}{|l|}{ 'Princess of India' nasturtium } \\
\hline 3 & $3.5 \mathrm{a}$ & $2.6 \mathrm{a}$ & $1.6 \mathrm{a}$ \\
\hline 5 & $3.7 \mathrm{a}$ & $3.1 \mathrm{a}$ & $2.0 \mathrm{a}$ \\
\hline 7 & $4.1 \mathrm{a}$ & $2.8 \mathrm{a}$ & $1.6 \mathrm{a}$ \\
\hline \multicolumn{4}{|l|}{ 'Kamome White' ornamental kale } \\
\hline 3 & $3.9 \mathrm{a}$ & $2.3 \mathrm{~b}$ & $2.1 \mathrm{a}$ \\
\hline 5 & $3.9 \mathrm{a}$ & $2.9 \mathrm{a}$ & $2.6 \mathrm{a}$ \\
\hline 7 & $4.3 \mathrm{a}$ & $2.9 \mathrm{a}$ & $2.3 \mathrm{a}$ \\
\hline \multicolumn{4}{|l|}{ 'Plush Salmon' petunia } \\
\hline 3 & $2.7 \mathrm{a}$ & $2.8 \mathrm{a}$ & $2.8 \mathrm{a}$ \\
\hline 5 & $3.4 \mathrm{a}$ & $2.3 \mathrm{a}$ & $2.9 \mathrm{a}$ \\
\hline 7 & $3.5 \mathrm{a}$ & $3.5 \mathrm{a}$ & $3.3 \mathrm{a}$ \\
\hline \multicolumn{4}{|l|}{ '21st Century Scarlet' phlox } \\
\hline 3 & $3.0 \mathrm{a}$ & $3.1 \mathrm{a}$ & $2.8 \mathrm{a}$ \\
\hline 5 & $3.3 \mathrm{a}$ & $4.3 \mathrm{a}$ & $3.8 \mathrm{a}$ \\
\hline 7 & $3.7 \mathrm{a}$ & $4.1 \mathrm{a}$ & $3.3 \mathrm{a}$ \\
\hline
\end{tabular}

(Continued on next page) 
Table 2. (Continued) Plant quality response of selected landscape-grown cool season annual species to nitrogen $(\mathrm{N})$ rate in 2 years in west-central Florida (U.S. Department of Agriculture hardiness zone $9 \mathrm{~b}$ ).

\begin{tabular}{|c|c|c|c|}
\hline \multirow{2}{*}{$\begin{array}{l}\text { Species and fertilizer } \mathrm{N} \text { rate } \\
\left(\mathrm{lb} / 1000 \mathrm{ft}^{2}\right)^{\mathrm{z}}\end{array}$} & \multicolumn{3}{|c|}{ Plant aesthetic quality $(0-5 \text { scale })^{\mathrm{y}}$} \\
\hline & $6 \mathrm{WAP}^{\mathrm{x}}$ & $12 \mathrm{WAP}$ & $18 \mathrm{WAP}$ \\
\hline \multicolumn{4}{|l|}{ 'White Breeze' shasta daisy } \\
\hline 3 & $3.6 \mathrm{a}$ & $2.9 \mathrm{a}$ & $3.2 \mathrm{a}$ \\
\hline 5 & $4.0 \mathrm{a}$ & $3.2 \mathrm{a}$ & $3.6 \mathrm{a}$ \\
\hline 7 & $3.9 \mathrm{a}$ & $3.5 \mathrm{a}$ & $3.3 \mathrm{a}$ \\
\hline \multicolumn{4}{|l|}{ 'Montego Yellow' /'Montego } \\
\hline \multicolumn{4}{|l|}{ Purple' snapdragon } \\
\hline 3 & $3.3 \mathrm{~b}$ & $3.1 \mathrm{~b}$ & $3.2 \mathrm{a}$ \\
\hline 5 & $3.3 \mathrm{~b}$ & $3.6 \mathrm{ab}$ & $3.8 \mathrm{a}$ \\
\hline 7 & $4.1 \mathrm{a}$ & $3.8 \mathrm{a}$ & $3.9 \mathrm{a}$ \\
\hline \multicolumn{4}{|l|}{ 'Fortress Dark Blue' statice } \\
\hline 3 & $3.0 \mathrm{a}$ & $2.5 \mathrm{a}$ & $2.1 \mathrm{a}$ \\
\hline 5 & $3.0 \mathrm{a}$ & $2.6 \mathrm{a}$ & $2.3 \mathrm{a}$ \\
\hline 7 & $3.0 \mathrm{a}$ & $2.3 \mathrm{a}$ & $1.7 \mathrm{a}$ \\
\hline \multicolumn{4}{|l|}{ 'Penny Blue' viola } \\
\hline 3 & $3.5 \mathrm{a}$ & $3.1 \mathrm{a}$ & $2.6 \mathrm{a}$ \\
\hline 5 & $3.7 \mathrm{a}$ & $3.6 \mathrm{a}$ & $3.6 \mathrm{a}$ \\
\hline 7 & $3.6 \mathrm{a}$ & $3.6 \mathrm{a}$ & $2.7 \mathrm{a}$ \\
\hline
\end{tabular}

${ }^{\mathrm{z}} 1 \mathrm{lb} / 1000 \mathrm{ft}^{2}=48.8243 \mathrm{~kg} \cdot \mathrm{ha}^{-1}$.

${ }^{\mathrm{y}} 0=$ dead plant; 5 = outstanding plant quality (dense leaf canopy, high-quality flowers, and no nutrient deficiencies or dieback).

"Weeks after planting.

"Mean separation for each species and WAP by Tukey's honestly significant difference test at $P<0.05$.

'Data not available.

Table 3. Plant quality response of selected landscape-grown warm-season annual species to nitrogen $(\mathrm{N})$ rate in 2 years in west-central Florida (U.S. Department of Agriculture hardiness zone 9b).

\begin{tabular}{|c|c|c|c|}
\hline \multirow{2}{*}{$\begin{array}{l}\text { Species and fertilizer } N \text { rate } \\
\left(1 \mathrm{~b} / 1000 \mathrm{ft}^{2}\right)^{\mathrm{z}}\end{array}$} & \multicolumn{3}{|c|}{ Plant aesthetic quality $(0-5 \text { scale })^{\mathrm{y}}$} \\
\hline & $6 \mathrm{WAP}^{\mathrm{x}}$ & 12 WAP & 18 WAP \\
\hline \multicolumn{4}{|l|}{ 'Lavender Shades'TM african daisy } \\
\hline 3 & $3.8 \mathrm{a}$ & $3.3 \mathrm{a}$ & $3.1 \mathrm{a}$ \\
\hline 5 & $3.9 \mathrm{a}$ & $3.9 \mathrm{a}$ & $3.2 \mathrm{a}$ \\
\hline 7 & $3.8 \mathrm{a}$ & $3.8 \mathrm{a}$ & $3.0 \mathrm{a}$ \\
\hline \multicolumn{4}{|c|}{$\begin{array}{l}\text { 'Blue Puffs Improved (Blue Danube)' } \\
\text { ageratum }\end{array}$} \\
\hline 3 & $2.6 \mathrm{a}^{\mathrm{w}}$ & $1.4 \mathrm{a}$ & $1.5 \mathrm{a}$ \\
\hline 5 & $3.1 \mathrm{a}$ & $1.2 \mathrm{a}$ & $1.8 \mathrm{a}$ \\
\hline 7 & $2.8 \mathrm{a}$ & $2.3 \mathrm{a}$ & $2.3 \mathrm{a}$ \\
\hline \multicolumn{4}{|l|}{ 'Lavender Pink'TM angelonia } \\
\hline 3 & $4.6 \mathrm{a}$ & $3.3 \mathrm{a}$ & $4.8 \mathrm{a}$ \\
\hline 5 & $4.5 \mathrm{a}$ & $3.5 \mathrm{a}$ & $5.0 \mathrm{a}$ \\
\hline 7 & $4.7 \mathrm{a}$ & $4.2 \mathrm{a}$ & $5.0 \mathrm{a}$ \\
\hline \multicolumn{4}{|l|}{ 'Komachi' balloon flower } \\
\hline 3 & $3.8 \mathrm{a}$ & $3.2 \mathrm{a}$ & $2.1 \mathrm{a}$ \\
\hline 5 & $3.9 \mathrm{a}$ & $2.9 \mathrm{a}$ & $2.2 \mathrm{a}$ \\
\hline 7 & $4.2 \mathrm{a}$ & $2.9 \mathrm{a}$ & $2.0 \mathrm{a}$ \\
\hline \multicolumn{4}{|l|}{ 'Spiky Purple' celosia } \\
\hline 3 & $2.9 \mathrm{a}$ & $0.0 \mathrm{a}$ & $0.0 \mathrm{a}$ \\
\hline 5 & $3.0 \mathrm{a}$ & $0.0 \mathrm{a}$ & $0.0 \mathrm{a}$ \\
\hline 7 & $2.8 \mathrm{a}$ & $0.0 \mathrm{a}$ & $0.0 \mathrm{a}$ \\
\hline \multicolumn{4}{|l|}{$\begin{array}{l}\text { 'Cosmic Mix' / Cosmic Orange’ } \\
\text { cosmos }\end{array}$} \\
\hline 3 & $1.7 \mathrm{a}$ & $0.0 \mathrm{a}$ & $0.0 \mathrm{a}$ \\
\hline 5 & $1.7 \mathrm{a}$ & $0.0 \mathrm{a}$ & $0.0 \mathrm{a}$ \\
\hline 7 & $1.2 \mathrm{a}$ & $0.0 \mathrm{a}$ & $0.0 \mathrm{a}$ \\
\hline
\end{tabular}

(Continued on next page)
Nitrogen fertilizer rates did not affect the aesthetic quality of warm-season annual species, with the exception of ornamental basil (Ocimum basilicum) (Table 3). Ornamental basil produced higher quality plants when supplied with $\mathrm{N}$ at the annual rate of $5 \mathrm{lb} / 1000 \mathrm{ft}^{2}$ compared with plants receiving the $3 \mathrm{lb} / 1000 \mathrm{ft}^{2}$ annual $\mathrm{N}$ rate at $6 \mathrm{WAP}$ or the $7 \mathrm{lb} / 1000 \mathrm{ft}^{2}$ annual $\mathrm{N}$ rate at 12 WAP. However, aesthetic quality of ornamental basil was unaffected by $\mathrm{N}$ rate at 18 WAP. Warm-season annual quality ratings ranged from 0.0 to 4.7 when plants were fertilized at the $3 \mathrm{lb} / 1000 \mathrm{ft}^{2}$ annual $\mathrm{N}$ rate. The quality of several species [e.g., celosia, (Celosia spicata), cosmos (Cosmos sulphureus), lavender (Lavandula angustifolia), nicotiana (Nicotiana alata), niermbergia (Nierembergia hippomanica), and salvia (Saliva splendens)] was below average (or plants were dead) toward the end of the evaluation period, regardless of $\mathrm{N}$ fertilizer rates (Table 3 ). We suspect that other factors, such as species selection, growing environment, or plant age, contributed to the low quality or death of these warm-season annual species.

Wright et al. (2009) reported that growth and dry weight of four annual landscape plants ['Cocktail Vodka' begonia (Begonia xsemperflorenscultorum), 'Red Hot Sally' salvia, 'Bonanza Yellow' marigold (Tagetes erecta), and 'Cooler Pink' vinca (Catharanthus roseus] increased as $\mathrm{N}$ fertilizer rate increased from 0 to $2 \mathrm{lb} / 1000 \mathrm{ft}^{2} \mathrm{~N}$ per year; but, the authors reported no effect of $\mathrm{N}$ rate on aesthetic quality. However, their study was conducted in Virginia and may not be directly comparable to central Florida. Shurberg et al. (2012b) reported that the application of $>8 \mathrm{lb} / 1000 \mathrm{ft}^{2}$ of $\mathrm{N}$ annually in central Florida was required to maximize size index and dry biomass of six species of annual landscape plants ['Profusion Cherry' zinnia, 'Cora White' vinca, 'Golden Globe' melampodium (Melampodium divaricatum), 'Telstar Crimson' dianthus (Dianthus chinensis), 'Delta Pure Violet' pansy (Viola wittrockiana), and 'Montego Yellow' snapdragon]; however, acceptable aesthetic quality was achieved with much lower $\mathrm{N}$ rates of $\left(2\right.$ to $6 \mathrm{lb} / 1000 \mathrm{ft}^{2} \mathrm{~N}$ per year), depending on species.

Perennials. As reported for the annual species, the aesthetic quality 
Table 3. (Continued) Plant quality response of selected landscape-grown warmseason annual species to nitrogen $(\mathrm{N})$ rate in 2 years in west-central Florida (U.S. Department of Agriculture hardiness zone $9 \mathrm{~b}$ ).

\begin{tabular}{|c|c|c|c|}
\hline \multirow{2}{*}{$\begin{array}{l}\text { Species and fertilizer } N \text { rate } \\
\left(1 \mathrm{~b} / 1000 \mathrm{ft}^{2}\right)^{\mathrm{z}}\end{array}$} & \multicolumn{3}{|c|}{ Plant aesthetic quality $(0-5 \text { scale })^{\mathrm{y}}$} \\
\hline & $6 \mathrm{WAP}^{\mathrm{x}}$ & 12 WAP & 18 WAP \\
\hline \multicolumn{4}{|l|}{ 'Silverdust' dusty miller } \\
\hline 3 & $4.7 \mathrm{a}$ & $4.0 \mathrm{a}$ & $4.0 \mathrm{a}$ \\
\hline 5 & $4.3 \mathrm{a}$ & $3.5 \mathrm{a}$ & $3.8 \mathrm{a}$ \\
\hline 7 & $4.5 \mathrm{a}$ & $3.8 \mathrm{a}$ & $3.6 \mathrm{a}$ \\
\hline \multicolumn{4}{|l|}{ 'Kiss Orange Flame' gazania } \\
\hline 3 & $3.1 \mathrm{a}$ & $2.4 \mathrm{a}$ & $1.9 \mathrm{a}$ \\
\hline 5 & $4.0 \mathrm{a}$ & $3.1 \mathrm{a}$ & $1.9 \mathrm{a}$ \\
\hline 7 & $3.8 \mathrm{a}$ & $2.7 \mathrm{a}$ & $2.3 \mathrm{a}$ \\
\hline \multicolumn{4}{|l|}{ 'Lavender Lady' lavender } \\
\hline 3 & $3.3 \mathrm{a}$ & $1.2 \mathrm{a}$ & $0.0 \mathrm{a}$ \\
\hline 5 & $3.8 \mathrm{a}$ & $1.6 \mathrm{a}$ & $0.9 \mathrm{a}$ \\
\hline 7 & $3.6 \mathrm{a}$ & $2.1 \mathrm{a}$ & $1.2 \mathrm{a}$ \\
\hline \multicolumn{4}{|l|}{ 'Riverine Sky Blue' lobelia } \\
\hline 3 & $2.6 \mathrm{a}$ & $1.2 \mathrm{a}$ & $0.0 \mathrm{a}$ \\
\hline 5 & $2.8 \mathrm{a}$ & $1.5 \mathrm{a}$ & $0.0 \mathrm{a}$ \\
\hline 7 & $2.6 \mathrm{a}$ & $0.8 \mathrm{a}$ & $0.0 \mathrm{a}$ \\
\hline \multicolumn{4}{|l|}{ 'Bonanza Orange' marigold } \\
\hline 3 & $3.6 \mathrm{a}$ & $1.2 \mathrm{a}$ & $1.5 \mathrm{a}$ \\
\hline 5 & $3.4 \mathrm{a}$ & $1.0 \mathrm{a}$ & $1.5 \mathrm{a}$ \\
\hline 7 & $3.7 \mathrm{a}$ & $1.1 \mathrm{a}$ & $1.7 \mathrm{a}$ \\
\hline \multicolumn{4}{|l|}{ 'Sundial Yellow' moss rose } \\
\hline 3 & $2.8 \mathrm{a}$ & $1.4 \mathrm{a}$ & $2.3 \mathrm{a}$ \\
\hline 5 & $3.3 \mathrm{a}$ & $1.6 \mathrm{a}$ & $2.5 \mathrm{a}$ \\
\hline 7 & $3.6 \mathrm{a}$ & $2.6 \mathrm{a}$ & $2.5 \mathrm{a}$ \\
\hline \multicolumn{4}{|l|}{ 'Saratoga Red' nicotiana } \\
\hline 3 & $2.0 \mathrm{a}$ & $0.0 \mathrm{a}$ & $0.0 \mathrm{a}$ \\
\hline 5 & $2.3 \mathrm{a}$ & $0.0 \mathrm{a}$ & $0.0 \mathrm{a}$ \\
\hline 7 & $1.7 \mathrm{a}$ & $0.0 \mathrm{a}$ & $0.0 \mathrm{a}$ \\
\hline \multicolumn{4}{|l|}{ 'White Robe' nierembergia } \\
\hline 3 & $3.3 \mathrm{a}$ & $2.1 \mathrm{a}$ & $1.5 \mathrm{a}$ \\
\hline 5 & $3.4 \mathrm{a}$ & $1.8 \mathrm{a}$ & $0.6 \mathrm{a}$ \\
\hline 7 & $3.5 \mathrm{a}$ & $2.3 \mathrm{a}$ & $1.3 \mathrm{a}$ \\
\hline \multicolumn{4}{|c|}{ 'Cardinal Organic' ornamental basil } \\
\hline 3 & $3.3 \mathrm{~b}$ & $2.9 \mathrm{ab}$ & $2.7 \mathrm{a}$ \\
\hline 5 & $4.2 \mathrm{a}$ & $3.1 \mathrm{a}$ & $2.6 \mathrm{a}$ \\
\hline 7 & $3.9 \mathrm{ab}$ & $2.8 \mathrm{~b}$ & $2.6 \mathrm{a}$ \\
\hline \multicolumn{4}{|l|}{ 'Medusa' ornamental pepper } \\
\hline 3 & $3.8 \mathrm{a}$ & $2.2 \mathrm{a}$ & $1.8 \mathrm{a}$ \\
\hline 5 & $4.0 \mathrm{a}$ & $1.8 \mathrm{a}$ & $1.5 \mathrm{a}$ \\
\hline 7 & $4.0 \mathrm{a}$ & $1.7 \mathrm{a}$ & $1.7 \mathrm{a}$ \\
\hline \multicolumn{4}{|l|}{ 'Vista Red' salvia } \\
\hline 3 & $2.3 \mathrm{a}$ & $0.6 \mathrm{a}$ & $0.0 \mathrm{a}$ \\
\hline 5 & $2.5 \mathrm{a}$ & $0.5 \mathrm{a}$ & $0.0 \mathrm{a}$ \\
\hline 7 & 2. a & $0.8 \mathrm{a}$ & $0.0 \mathrm{a}$ \\
\hline \multicolumn{4}{|l|}{ 'Crown Plum' torenia } \\
\hline 3 & $3.2 \mathrm{a}$ & $2.5 \mathrm{a}$ & $2.3 \mathrm{a}$ \\
\hline 5 & $3.2 \mathrm{a}$ & $2.7 \mathrm{a}$ & $2.8 \mathrm{a}$ \\
\hline 7 & $3.0 \mathrm{a}$ & $2.3 \mathrm{a}$ & $1.8 \mathrm{a}$ \\
\hline \multicolumn{4}{|l|}{ 'Profusion Cherry' zinnia } \\
\hline 3 & $3.8 \mathrm{a}$ & $2.2 \mathrm{a}$ & $2.3 \mathrm{a}$ \\
\hline 5 & $3.8 \mathrm{a}$ & $2.1 \mathrm{a}$ & $2.7 \mathrm{a}$ \\
\hline 7 & $3.8 \mathrm{a}$ & $2.2 \mathrm{a}$ & $2.6 \mathrm{a}$ \\
\hline
\end{tabular}

${ }^{\mathrm{z}} \mathrm{l} \mathrm{lb} / 1000 \mathrm{ft}^{2}=48.8243 \mathrm{~kg} \cdot \mathrm{ha}^{-1}$

${ }^{\mathrm{y}} 0=$ dead plant; 5 = outstanding plant quality (dense leaf canopy, high-quality flowers, and no nutrient deficiencies or dieback).

'Weeks after planting.

"Mean separation for each species and WAP by Tukey's honestly significant difference test at $P<0.05$. ratings of few perennial species were affected by $\mathrm{N}$ rate (Table 4). Again the goal was to validate $\mathrm{N}$ fertilizer rates determined in previous work by Shurberg et al. (2012a) on perennial plant growth to $\mathrm{N}$ fertilization rate. Differences in the aesthetic quality of perennial species due to $\mathrm{N}$ rate treatments were limited to blanket flower (18 WAP) and salvia [54 WAP (Table 4)]. Higher quality blanket flower and salvia plants were produced when plants received 3 or $5 \mathrm{lb} / 1000 \mathrm{ft}^{2} \mathrm{~N}$ annually compared with plants fertilized with $\mathrm{N}$ at the $1 \mathrm{lb} / 1000 \mathrm{ft}^{2}$ per year rate (Table 4 ).

Chen et al. (2011) reported that application of $\mathrm{N}$ fertilizer improved both growth and visual quality of six perennial landscape species in Louisiana up to a rate of $2 \mathrm{lb} / 1000 \mathrm{ft}^{2} \mathrm{~N}$ per year. Nitrogen fertilizer supplied at the higher rate of $4 \mathrm{lb} / 1000 \mathrm{ft}^{2} \mathrm{~N}$ per year continued to push growth of cigar plant (Cuphea ignea), 'New Gold' lantana (Lantana $\times$ hybrid), and 'Goldsturm' rudbeckia (Rudbeckia fulgida), but did not affect visual quality. However, results might vary in central Florida. Shurberg et al. (2012a) found that higher rates of $\mathrm{N}$ fertilization in Florida $\left(>8 \mathrm{lb} / 1000 \mathrm{ft}^{2}\right.$ $\mathrm{N}$ per year) could increase growth of five perennial landscape species [bush daisy (Gamolepis chrysanthemoides), 'New Gold' lantana and 'Mystic Spires' salvia, 'Evergreen Giant' liriope (Liriope muscari), and 'White Christmas' caladium (Caladium bicolor)], but that acceptable aesthetic quality was achieved at low rates of 0 to $4 \mathrm{lb} /$ $1000 \mathrm{ft}^{2} \mathrm{~N}$ per year, depending on species.

\section{Conclusions}

While the aesthetic quality of some evaluated landscape-grown annual and perennial species did respond to higher fertilizer rates, our results showed that application of a controlled-release fertilizer at an $\mathrm{N}$ rate of $1 \mathrm{lb} / 1000 \mathrm{ft}^{2}$ annually (perennials) to $3 \mathrm{lb} / 1000 \mathrm{ft}^{2}$ annually (annuals) was adequate to sustain acceptable quality for most species. Increasing $\mathrm{N}$ fertilizer rate did not improve plant quality for species with quality ratings that were less than acceptable $(<3)$, suggesting that other factors (e.g., environment, species selection, or plant age) other than $\mathrm{N}$ fertilization might have affected plant quality. Based on these results, we suggest that an $\mathrm{N}$ 
Table 4. Plant quality response of selected landscape-grown herbaceous perennial species planted 4 Mar. 2011 to nitrogen (N) rate in 2 years in westcentral Florida (U.S. Department of Agriculture hardiness zone 9b).

\begin{tabular}{|c|c|c|c|c|}
\hline \multirow[b]{2}{*}{$\begin{array}{l}\text { WAP and fertilizer } \\
\mathrm{N} \text { rate }\left(\mathrm{lb} / 1000 \mathrm{ft}^{2}\right)^{\mathrm{z}}\end{array}$} & \multicolumn{4}{|c|}{ Plant aesthetic quality $(0-5 \text { scale })^{y}$} \\
\hline & $\begin{array}{l}\text { Blanket } \\
\text { flower }\end{array}$ & Goldenrod & $\begin{array}{c}\text { Mondo } \\
\text { grass }\end{array}$ & $\begin{array}{c}\text { 'Mystic } \\
\text { Spires' Salvia }\end{array}$ \\
\hline \multicolumn{5}{|l|}{$6 \mathrm{WAP}$} \\
\hline 1 & $3.6 \mathrm{a}^{\mathrm{x}}$ & $3.2 \mathrm{a}$ & $3.4 \mathrm{a}$ & $3.7 \mathrm{a}$ \\
\hline 3 & $3.9 \mathrm{a}$ & $3.2 \mathrm{a}$ & $3.4 \mathrm{a}$ & $3.8 \mathrm{a}$ \\
\hline 5 & $3.8 \mathrm{a}$ & $3.1 \mathrm{a}$ & $3.3 \mathrm{a}$ & $4.3 \mathrm{a}$ \\
\hline \multicolumn{5}{|l|}{12 WAP } \\
\hline 1 & $2.9 \mathrm{a}$ & $2.9 \mathrm{a}$ & $3.2 \mathrm{a}$ & $2.6 \mathrm{a}$ \\
\hline 3 & $3.2 \mathrm{a}$ & $3.1 \mathrm{a}$ & $3.2 \mathrm{a}$ & $2.9 \mathrm{a}$ \\
\hline 5 & $3.0 \mathrm{a}$ & $3.3 \mathrm{a}$ & $2.9 \mathrm{a}$ & $3.1 \mathrm{a}$ \\
\hline \multicolumn{5}{|l|}{18 WAP } \\
\hline 1 & $3.5 \mathrm{~b}$ & $2.8 \mathrm{a}$ & $2.2 \mathrm{a}$ & $2.8 \mathrm{a}$ \\
\hline 3 & $3.6 \mathrm{a}$ & $3.0 \mathrm{a}$ & $2.2 \mathrm{a}$ & $3.1 \mathrm{a}$ \\
\hline 5 & $4.0 \mathrm{a}$ & $3.1 \mathrm{a}$ & $1.8 \mathrm{a}$ & $3.2 \mathrm{a}$ \\
\hline \multicolumn{5}{|l|}{$24 \mathrm{WAP}$} \\
\hline 1 & $-^{w}$ & $3.6 \mathrm{a}$ & $2.4 \mathrm{a}$ & $2.9 \mathrm{a}$ \\
\hline 3 & - & $4.0 \mathrm{a}$ & $2.3 \mathrm{a}$ & $3.1 \mathrm{a}$ \\
\hline 5 & - & $3.9 \mathrm{a}$ & $2.0 \mathrm{a}$ & $3.1 \mathrm{a}$ \\
\hline \multicolumn{5}{|l|}{$30 \mathrm{WAP}$} \\
\hline 1 & $2.5 \mathrm{a}$ & $2.7 \mathrm{a}$ & $2.3 \mathrm{a}$ & $1.9 \mathrm{a}$ \\
\hline 3 & $2.9 \mathrm{a}$ & $2.6 \mathrm{a}$ & $2.4 \mathrm{a}$ & $2.2 \mathrm{a}$ \\
\hline 5 & $2.8 \mathrm{a}$ & $2.7 \mathrm{a}$ & $2.2 \mathrm{a}$ & $2.4 \mathrm{a}$ \\
\hline \multicolumn{5}{|l|}{$36 \mathrm{WAP}$} \\
\hline 1 & $3.1 \mathrm{a}$ & $1.1 \mathrm{a}$ & $2.4 \mathrm{a}$ & $2.0 \mathrm{a}$ \\
\hline 3 & $3.2 \mathrm{a}$ & $1.0 \mathrm{a}$ & $2.4 \mathrm{a}$ & $2.1 \mathrm{a}$ \\
\hline 5 & $3.0 \mathrm{a}$ & $1.0 \mathrm{a}$ & $2.4 \mathrm{a}$ & $2.4 \mathrm{a}$ \\
\hline \multicolumn{5}{|l|}{42 WAP } \\
\hline 1 & $1.1 \mathrm{a}$ & $1.1 \mathrm{a}$ & $2.1 \mathrm{a}$ & $2.6 \mathrm{a}$ \\
\hline 3 & $1.1 \mathrm{a}$ & $1.3 \mathrm{a}$ & $2.3 \mathrm{a}$ & $2.7 \mathrm{a}$ \\
\hline 5 & $1.0 \mathrm{a}$ & $1.2 \mathrm{a}$ & $2.1 \mathrm{a}$ & $2.8 \mathrm{a}$ \\
\hline \multicolumn{5}{|l|}{48 WAP } \\
\hline 1 & $2.1 \mathrm{a}$ & $1.8 \mathrm{a}$ & $2.1 \mathrm{a}$ & $2.2 \mathrm{a}$ \\
\hline 3 & $2.2 \mathrm{a}$ & $2.0 \mathrm{a}$ & $2.3 \mathrm{a}$ & $2.3 \mathrm{a}$ \\
\hline 5 & $2.1 \mathrm{a}$ & $1.9 \mathrm{a}$ & $1.9 \mathrm{a}$ & $2.3 \mathrm{a}$ \\
\hline \multicolumn{5}{|l|}{$54 \mathrm{WAP}$} \\
\hline 1 & $2.5 \mathrm{a}$ & $2.7 \mathrm{a}$ & $1.9 \mathrm{a}$ & $1.6 \mathrm{~b}$ \\
\hline 3 & $2.6 \mathrm{a}$ & $2.6 \mathrm{a}$ & $1.9 \mathrm{a}$ & $2.2 \mathrm{a}$ \\
\hline 5 & $2.8 \mathrm{a}$ & $2.7 \mathrm{a}$ & $1.8 \mathrm{a}$ & $2.6 \mathrm{a}$ \\
\hline
\end{tabular}

${ }^{2} \mathrm{WAP}=$ week after planting; $1 \mathrm{lb} / 1000 \mathrm{ft}^{2}=48.8243 \mathrm{~kg} \cdot \mathrm{ha}^{-1}$.

${ }^{y} 0=$ dead plant; $5=$ outstanding plant quality (dense leaf canopy, high-quality flowers, and no nutrient deficiencies or dieback).

'Mean separation for each species and WAP by Tukey's honestly significant difference test at $P<0.05$.

"Data not available.

fertilizer recommendation of 2 to 4 $\mathrm{lb} / \mathrm{l} 000 \mathrm{ft}^{2}$ per year of controlledrelease fertilizer, as reported by Shurberg et al. $(2012 \mathrm{a}, \mathrm{b})$ should be adequate for maintaining acceptable quality annual and herbaceous perennial plant species when grown in the landscape in west-central Florida. However, quality response may vary with the location and soil type, type of species, year planted, and type of $\mathrm{N}$ fertilizer product. Future work should address the environmental benefits of lower $\mathrm{N}$ rates and changes in fertilizer application methods as well as the economic benefit of using lower $\mathrm{N}$ rates. However, the purpose of this work was to provide science-based evidence for revising the FFL-IFAS $\mathrm{N}$ fertilizer recommendations for annual and perennial plants.

\section{Literature cited}

Chen, Y., R.P. Bracy, A.D. Owings, and J.P. Quebedeaux. 2011. Controlledrelease fertilizer type and rate affect landscape establishment of seven herbaceous perennials. HortTechnology 21:336342.
Florida Department of Environmental Protection. 2002. Best management practices for protection of water resources in Florida. Florida Dept. Environ. Protection, Tallahassee, FL.

Florida Friendly Landscaping Program. 2009. The Florida Yards \& Neighborhoods handbook. Univ. Florida Inst. Food Agr. Sci., Gainesville, FL.

Latimer, J.G., S.K. Braman, R.B. Beverly, P.A. Thomas, J.T. Walker, B. Sparks, R.D. Oetting, J.M. Ruter, W. Florkowski, D.L. Olson, C.D. Robacker, M.P. Garber, O.M. Lindstrom, and W.G. Hudson. 1996. Reducing the pollution potential of pesticides and fertilizers in the environmental horticulture industry: II. Lawn care and landscape management. HortTechnology 6:222-232.

Line, D.E., N.M. White, D.L. Osmond, G.D. Jennings, and C.B. Mojonnier. 2002. Pollutant export from various land uses in the upper neuse river basin. Water Environ. Res. 74:100-108.

Matson, P.A., W.J. Parton, A.G. Power, and M.J. Swift. 1997. Agricultural intensification and ecosystem properties. Science 277:504-509.

Shober, A.L., S. Davis, M.D. Dukes, G.C. Denny, S.P. Brown, and S. Vyapari. 2009. Performance of Florida landscape plants when irrigated by ET-based controllers and time-based methods. J. Environ. Hort. 27:251-256.

Shurberg, G., A.L. Shober, C. Wiese, G. Denny, G.W. Knox, K.A. Moore, and M.C. Giurcanu. 2012a. Growth and quality response of five landscape-grown herbaceous perennials to nitrogen fertilization at five rates. Hort Technology 22:787-797.

Shurberg, G., A.L. Shober, C. Wiese, G. Denny, G.W. Knox, K.A. Moore, and M.C. Giurcanu. 2012b. Response of landscape-grown warm- and cool-season annuals to nitrogen fertilization at five rates. HortTechnology 22:368-375.

University of Florida. 2014. Florida automated weather network, report generator. 5 Aug. 2014. <http://fawn.ifas.ufl.edu/ data/reports/>.

U.S. Department of Agriculture. 2004. Official soil series descriptions. 11 Nov. 2011. <http://soils.usda.gov/technical/ classification/osd/index.html>.

Wright, R.D., B.E. Jackson, M.C. Barnes, and J.F. Browder. 2009. The landscape performance of annual bedding plants grown in pine tree substrate. HortTechnology 19:78-82. 